\title{
Eosinophilic peritonitis
}

Singapore Med J 2019; 60(11): 605 https://doi.org/10.11622/smedj.2019149

Dear Sir,

Peritoneal dialysis (PD) peritonitis is typically caused by gram-positive bacteria but may be culture negative. Culture-negative peritonitis is often caused by fastidious organisms. Infective peritonitis is a common complication of PD, with an incidence of 42 cases per 100 patient-years, ${ }^{(1)}$ while eosinophilic peritonitis is a rare and poorly described non-infective entity that is often missed or confused for bacterial PD peritonitis. We herein report a case of eosinophilic peritonitis that was treated successfully.

A 44-year-old man presented with constant abdominal pain since commencing automated PD one week earlier. A Tenckhoff catheter had been inserted five weeks before commencing PD. The pain was worst when draining at the end of PD exchanges and sleep was disrupted. He also described cloudy effluent of several days' duration. The patient denied any other symptoms. He had a background of Fabry disease with end-stage renal failure.

The patient's full blood count was abnormal only for eosinophil count, with eosinophilia of $0.75 \times 10^{9} \mathrm{mg} / \mathrm{L}$ (normal $<0.60 \times$ $10^{9} \mathrm{mg} / \mathrm{L}$ ). His C-reactive protein was $8 \mathrm{mg} / \mathrm{L}$ (normal range $<5 \mathrm{mg} / \mathrm{L}$ ). PD fluid microscopy revealed a white blood cell count of $797 \times 10^{6} / \mathrm{L}$ with $12 \%$ polymorphs and $49 \%$ eosinophils, with no microbial growth. The patient was commenced empirically on intraperitoneal antibiotics for presumed bacterial PD peritonitis. He reported no improvement in pain after one week of treatment. Repeat PD fluid examination showed worsening to a white blood cell count of 1,490 × 10\%/L, with 35\% eosinophils, 56\% mononuclears and $6 \%$ polymorphs.

Antibiotics were ceased. The patient was started on prednisone $25 \mathrm{mg}$ daily with rapid improvement in symptoms. After one week, further PD fluid analysis demonstrated near resolution of cell counts. The white blood cell count decremented to $55 \times 10^{6} / \mathrm{L}$, and the fluid cultures remained negative. Prednisone was stopped after a ten-day course, and the patient experienced no further symptoms. The diagnosis of eosinophilic peritonitis was made. The patient continued uneventfully on automated PD to the three-month follow-up.

Eosinophilic peritonitis is a non-infectious, presumably allergic, inflammatory response to PD apparatus. The true incidence is unknown but is certainly very low. Nonetheless, it remains a relevant entity, particularly in Asian dialysis cohorts, where the uptake of PD is high.(2) Proposed definitions include abdominal pain or cloudy peritoneal dialysate coupled with either a total perineal fluid eosinophil count greater than 100 cells $/ \mathrm{mm}^{3}$ or a fluid eosinophil count greater than $40 \mathrm{cells} / \mathrm{mm}^{3}$, where eosinophils comprise at least $10 \%$ of the peritoneal fluid white blood cell count. ${ }^{(3)}$ Most cases occur within one month of insertion of the peritoneal catheter, and the commonest symptoms are abdominal pain, cloudy effluent or catheter dysfunction. There is no evidence-based treatment. Patients in whom these criteria overlap and who also meet the criteria for bacterial PD, such as where the absolute eosinophil count is very high but it nonetheless still accounts for only a small proportion of the cell differential, should be treated with standard, protocolised antibiotics. ${ }^{(4)}$ Case series for pure eosinophilic peritonitis have trialled intraperitoneal hydrocortisone, oral prednisone and oral antihistamines, with varying success. The choice of agent, dose and duration is currently based on individual patient circumstances and physician preference. In patients who do not require early catheter removal due to intolerance, the condition should resolve within weeks or months.

Yours sincerely,

Julian $\underline{\text { Yaxley }}^{1}$, Alan $\underline{\text { Parnham }}^{1}$

${ }^{1}$ Department of Nephrology, Gold Coast University Hospital, Gold Coast, Queensland, Australia. julianyaxley@yahoo.com.au

\section{References}

1. Pulliam J, Li N, Maddux F, et al. First-year outcomes of incident peritoneal dialysis patients in the United States. Am J Kidney Dis 2014; 64:761-9.

2. Kwong VW, Li PK. Peritoneal dialysis in Asia. Kidney Dis (Basel) 2015; 1:147-56.

3. Ejaz AA, Fitzpatrick PM, Durkin AJ, et al. Pathophysiology of peritoneal fluid eosinophilia in peritoneal dialysis patients. Nephron 1999; 81:125-30.

4. Fontán M, Rodríguez-Carmona A, Galed I, et al. Incidence and significance of peritoneal eosinophilia during peritoneal dialysis-related peritonitis. Perit Dial Int 2003; 23:460-4. 